\title{
ASPECTOS DO SENSORIAMENTO REMOTO GEOLÓGICO NA BACIA DO ARARIPE (PE/CE/PI/BRASIL)
}

\author{
Aerson Moreira Barreto Junior ${ }^{1}$ \\ Admilson da Penha Pacheco ${ }^{2}$ \\ Maria Alcione Lima Celestino ${ }^{1}$ \\ 10.18190/1980-8208/estudosgeologicos.v28n2p82-98 \\ 1Programa de Pós-Graduação em Geociências - UFPE \\ aerson@hotmail.com; alcionelimma@hotmail.com \\ ${ }^{2}$ Departamento de Engenharia Cartográfica e Programa de Pós-graduação em Geociências \\ E-mail: pacheco3p@gmail.com
}

\section{RESUMO}

A Bacia do Sedimentar do Araripe é a maior dentre as bacias interiores do Nordeste do Brasil, posicionando-se sobre terrenos cristalinos pré-cambrianos, contendo rochas magmáticas e metamórficas, litologias pertencentes à Zona Transversal da Província Borborema, a sul do Lineamento Patos e a norte do Lineamento Pernambuco. Paralelamente à evolução dos sistemas de aquisição de dados de sensoriamento remoto, surgiram novas abordagens metodológicas em processamento digital de imagens e melhorias em metodologias já existentes, otimizando assim a extração de informações a partir imagens orbitais e aéreas. A integração de dados de sensoriamento remoto óptico e de radar tem favorecido diversos estudos geológicos desde a década de 1970. O uso das imagens de satélite e de radar tem se tornado cada vez mais frequente em estudos geológicos, hidrológicos, geomorfológicos, ecológicos, dentre outros, em particular para análises tanto quantitativas como qualitativas do relevo e seus agentes modificadores. As imagens do Satélite Landsat e dados da missão SRTM (Shuttle Radar Topography Mission), devido à disponibilidade gratuita e abrangência global, apresentam atualmente predominância nas diferentes aplicações relacionadas com mapeamentos geológicos. Estudos envolvendo sensoriamento remoto geológico tem sido realizado na Bacia do Araripe desde a década de 1960. Este trabalho tem como objetivo contextualizar o estado da arte do sensoriamento remoto geológico na Bacia do Araripe, caracterizando os seus subdomínios do conhecimento - geomorfológico, ambiental e hidrogeológico, a partir dos principais métodos e resultados encontrados nas publicações pré-selecionadas.

Palavras chave: Bacia do Araripe, Sensoriamento Remoto, Mapeamento Geológico, Landsat, Radar.

\begin{abstract}
The Araripe Basin is the biggest of the Brazil Northeast interior basins. This basin lies over Precambrian terrains of the Borborema Province, containing metamorphic and magmatic rocks related to the Transversal Fold Zone, between Patos and Pernambuco lineaments. At the same time as the data acquisition systems in remote sensing evolved, new methodological approaches in digital image processing and improvements in existing methods were developed, optimizing the data extraction from orbital and aerial images. The integration of optical and radar remote sensing data have favored many geological studies since the 1970s. Satellite and radar imaging have been applied more frequently in
\end{abstract}


matters of geology, hydrology, geomorphology, ecology, among others, mainly for the use of relief and its modifying agents quantitative and qualitative analysis. Due to the free availability and global coverage, the Landsat satellite imaging and the SRTM mission (Shuttle Radar Topography Mission) data are predominantly used in the different applications related to geological mapping. Studies involving remote sensing for geology purposes have been performed in the Araripe Basin since the 1960s. This paper aims to contextualize the geologic remote sensing state of the art in the Araripe Basin, characterizing its knowledge subdomains - geomorphological, environmental and hydrogeological, from the main methods and results found in the literature review.

Keywords: Araripe Basin, Remote Sensing, Geologic Mapping, Landsat, Radar.

\section{INTRODUÇÃO}

As técnicas de Sensoriamento Remoto tiveram nas últimas décadas uma grande evolução a partir do surgimento de modernos sistemas sensores, aéreos e orbitais, capazes de produzir imagens multi e hiperespectrais de alta resolução espacial, temporal e radiométrica, aumentando a capacidade de discriminar, cada vez melhor, os alvos da superfície terrestre e ampliando significativamente as aplicações relacionadas com mapeamentos geológicos. Paralelamente à evolução dos sistemas de aquisição de dados de Sensoriamento Remoto, surgiram novas abordagens metodológicas em processamento digital de imagens e melhorias em metodologias já existentes, otimizando assim a extração de informações a partir imagens orbitais e aéreas.

No que concerne a geologia e interpretação geológica, pode-se afirmar que a análise de imagens digitais do satélite Landsat e de radar da missão aeroespacial SRTM (Shuttle Radar Topography Mission) são ponto inicial para a cartografia geológica e, associadas a dados obtidos posteriormente em etapas de campo, auxiliam na confecção de mapas geológicos. Numa segunda fase ou em estudos espectrais específicos e complementares, a presença de limites entre objetos geologicamente distintos pode apresentar assinaturas espectrais diferentes, sendo marcadas por uma variação relativa entre níveis de cinza, podendo ainda ser realçadas com o uso de filtros (Meneses \& Almeida, 2012). A utilização de produtos integrados Landsat TM e aerogeofísicos gamaespectrométricos tem apresentado bons resultados na discriminação de unidades geológicas, principalmente em áreas pouco conhecidas geologicamente (Paradella, 1997). A vantagem da utilização de produtos integrados Landsat-aerogama-espectrométricos no mapeamento geoló-gico é que estes apresentam realces das feições estruturais de relevo e drenagem representadas na imagem Landsat, e também variações de matizes que representam as respostas gamaespectrométricas (Maducci et al., 2003).

No estudo das bacias sedimentares interiores, o uso de imagens de satélite mostra-se bastante eficaz na detecção e predição da ocorrência e intensidade de fraturas (Nelson, 2001). Abdullah et al. (2009), por exemplo, compararam bandas Landsat TM e imagens SPOT-5 no mapeamento automático de lineamentos em terreno composto de rochas sedimentares paleozoicas e mesozoicas, granitoides intrusivos e rochas vulcânicas, localizado no leste da Malásia, concluindo que a banda 4 do Landsat e a banda 3 do SPOT-5, foram mais eficientes, principalmente em áreas tropicais, bem como para extração manual de lineamentos (Conceição \& 
Silva, 2013). Os lineamentos, por exemplo, associados a quebras positivas (relacionadas a cristas de serras, escarpas, crateras) são delineados por pixels de tonalidade clara enquanto os lineamentos relativos às quebras negativas (referentes a juntas, falhas, zonas de cisalhamento, drenagem) aparecem na imagem como pixels alinhados de tons escuros (Abdullah et al., 2009).

O uso de dados de sensoriamento remoto, como os dados de modelos digitais de elevação (MDE/DEM) obtidos a partir de imagens orbitais, que possuem em seu conteúdo, informações relacionadas às feições geomorfológicas, podem representar a topografia ou a forma do terreno. Permitem, também, a derivação de uma série de produtos importantes na interpretação das formas de relevo de uma determinada área, configurando-se como uma importante ferramenta para análise e interpretação de estruturas geomorfológicas (Duarte et al., 2017). Os MDE podem ser aplicados na geomorfologia, na elaboração de mapas de concavidade e convexidade; na análise de rede hidrográfica, na delimitação automática de bacias, microbacias e áreas inundadas; animações; favorecendo a análise da área em estudo em diferentes ângulos, confecção de perfis topográficos e longitudinais (rios), além do uso para correção geométrica e radiométrica de imagens de sensoriamento remoto (Latrubesse et al., 2005; Carvalho \& Bayer, 2008).

O uso das imagens SRTM tem se tornado cada vez mais frequente em estudos geológicos, hidrológicos, geomorfológicos, geotécnicos, ecológicos, dentre outros, em particular para análises tanto quantitativas como qualitativas do relevo e seus agentes modificadores (Carvalho \& Latrubesse, 2004; Hoff et al., 2010; Silva, 2017), em especial na elaboração de mapas hipsométricos e clinográficos (declivi- dade), e de perfis topográficos, dentre outros produtos elaborados a partir de variáveis relacionadas à topografia (Carvalho \& Bayer, 2008). O emprego deste conjunto de produtos gerados a partir dos dados SRTM e a análise computacional destes dados, através de métodos automatizados desenvolvidos pela comunidade de usuários de dados de sensoriamento remoto, vem sendo amplamente explorado na produção de mapas interpretativos de relevo (Duarte et al., 2017).

As características da interferometria SAR (Radar de Abertura Sintética) possibilitam a elaboração de modelos digitais de elevação, que são importantes instrumentos no estudo da compartimentação de relevo (Silva \& Santos, 2007). Sendo, assim, de grande relevância para a compreensão da geologia de uma determinada região.

A fusão de imagens de sistemas ópticos e de radar permite combinar a resposta multiespectral do alvo, provinda do sistema óptico, com informações de relevo oriundas das imagens de radar (Souza Filho \& Paradella, 2002).

A combinação de imagens de satélite (sistema óptico) e de radar para a identificação de estruturas geológicas tem sido empregada por vários autores (Justo, 2006; Strugale et al., 2007, entre outros). O uso integrado destes produtos tem também favorecido o desenvolvimento da geologia costeira (Souza Filho \& Paradela, 2003; Restrepo \& Lope, 2008; Teixeira \& Souza Filho, 2009).

Grohmann et al. (2008) afirmam que a partir da análise de várias aplicações dos dados SRTM, torna-se possível o cálculo de variáveis topográficas, a identificação de formas de relevo e estruturas (potencialmente mascaradas sem imagens ópticas) e o cruzamento das informações altimétricas (e variáveis associadas) com dados de campo e de sensoriamento remoto óptico. Estas informações possibilitam a 
obtenção de dados topográficos de qualidade que podem ser aplicados em análise geomorfológica (Duarte et al., 2017).

A Bacia Sedimentar do Araripe é uma região onde as unidades geológicas se destacam pelo controle estrutural e litológico sobre a morfogênese, sendo o planalto sedimentar, em si, o seu compartimento geomorfológico mais conspícuo (Santos et al., 2009). Vários estudos científicos de sensoriamento remoto aplicado à geologia, com predominância de imagens de satélite e de radar, foram realizados na Bacia Sedimentar do Araripe nas últimas décadas. Este trabalho tem como objetivo contextualizar o estado da arte do sensoriamento remoto geológico na Bacia do Araripe, caracterizando os seus subdomínios do conhecimento geomorfológico, ambiental e hidrogeológico, a partir dos principais métodos $\mathrm{e}$ resultados encontrados nas publicações pré-selecionadas.

\section{BACIA SEDIMENTAR DO ARARIPE}

A Bacia do Araripe (Fig. 1) engloba partes dos estados de Pernambuco, Ceará e Piauí, no Nordeste brasileiro. Sua extensão de leste a oeste equivale a $225 \mathrm{~km}$ e de norte a sul a aproximadamente $75 \mathrm{~km}$ (Martill \& Wilby, 1993). No lado oriental, a altitude ultrapassa $950 \mathrm{~m}$, enquanto que nas proximidades da fronteira com o Piauí a altitude é de cerca de 700 m (Brito, 1979), sendo a maior dentre as bacias interiores do Nordeste do Brasil, possuindo aproximadamente 10.400 $\mathrm{km}^{2}$. (Carvalho \& Melo, 2012; Carvalho et al., 2012).

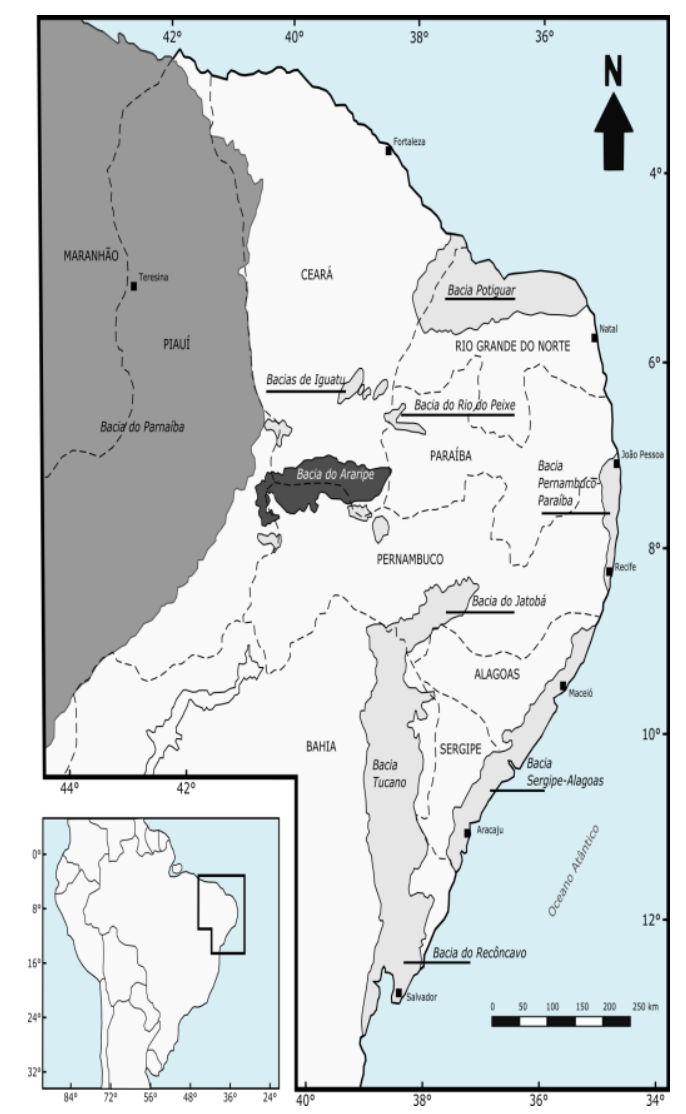

Figura 1 - Localização das bacias sedimentares do Nordeste brasileiro, com destaque para a Bacia do Araripe (em cinza escuro). Fonte: Modificado de Pires \& Guerra-Sommer (2011). 
A Bacia Sedimentar do Araripe repousa sobre terrenos cristalinos précambrianos, contendo rochas magmaticas e metamórficas, litologias pertencentes a diferentes domínios tectônicos do Sistema Orogênico Borborema (Delgado et. al., 2003). Os terrenos do embasamento já retrabalhados formam um conjunto de corpos lenticulares justapostos e sigmoides, separados por zonas de cisalhamento transcorrentes (Carvalho et al., 2012).

Alguns trabalhos postulam que a Bacia do Araripe foi formada pela reativação de estruturas do embasamento pré-cambriano devido à propagação de esforços tectônicos relacionados à fase rifte, no Eocretáceo, das bacias marginais brasileiras (Assine, 1990; Matos, 1992 e 1999; Ponte \& Ponte Filho, 1996, Dissertação Araripe).

A Chapada do Araripe é um planalto localizado na divisa dos estados brasileiros do Ceará, Piauí e Pernambuco. Ela abriga uma Floresta Nacional (1946 - Governo Federal o Decreto 9.226), uma Área de Proteção Ambiental (Criada em 04 de agosto de 1997) e um Geoparque (Desde 2006 integra a rede mundial de Geoparques). De acordo com Araújo et al. (2009), a vegetação é bastante diversificada, apresentando domínios de cerradão (tipo predominante), caatinga e cerrado. Muitas cidades ocupam áreas da chapada, provocando forte impacto no ambiente. Segundo o mesmo autor, parte considerável da mata original foi desmatada ou destruída por queimadas.

A Chapada do Araripe, uma superfície tabular fortemente influenciada pela estrutura geológica e pelo modelo de estratificação, e os patamares de entorno são constituídos por rochas de idade cretácica do Grupo Araripe, que se sobrepõem às rochas siluro-devonianas da Formação Cariri (Souza \& Oliveira, 2006).

O histórico tectônico estrutural dessa área tem a divisão do Pangeia, em especial o desmembramento da Gondwana em América do Sul e África, como principal evento causador de deformações crustais, principalmente pela formação do rifte Cariri-Potiguar, iniciado no Neocomiano e abortado no final do Barremiano (Claudino-Sales \& Pelvast, 2007). Entre o Jurássico e o Cretáceo Inferior (Barremiano), a região Araripe, que já arqueda, subsidiu mais ainda, em resposta aos esforços distensivos que preparavam a ruptura entre a América do Sul e a África, dando origem ao rifte intracontinental que se desenvolveu no local (Claudino-Sales \& Pelvast, 2007).

A bacia está dividida em três domínios geomorfológicos distintos (DNPM, 1996), a zona de chapada, a zona de talude e a zona de pediplano. A zona de chapada ou propriamente a Chapada do Araripe, tem relevo tipicamente tabular, com elevações em torno de 900 metros. A zona de talude é formada pela encosta que bordejam a zona de chapada e testemunhos da chapada. A zona de pediplano, possui ou apresenta relevo suave e pouco dissecado, caracterizado por morros alongados separados por vales amplos de fundo plano, com cotas médias de em torno de 400 metros. Dentro dessa unidade se encontra o Vale do Cariri (Aguiar, 2007).

A arquitetura interna da bacia pode ser descrita por dois compartimentos superpostos e de estilos estruturais distintos (Ponte \& Ponte Filho, 1996). O compartimento inferior, caracterizado por bacias do tipo rifte, divididas internamente por blocos estruturais (grábens e horsts), e o compartimento superior, representado pela cobertura tabular que constitui a chapada do Araripe (Veríssimo \& Aguiar, 2005). No compartimento inferior ocorre um conjunto de meio-grábens assimétricos, fragmentados por altos de embasamento e complexos sistemas de falhas normais?? e lineamentos E-W a NE-SW, 
associados ao trend estrutural do Província Borborema, e NW-SE, relacionados a processos extensionais cuja evolução estaria associada ao riftvalley na extremidade norte do Aulacógeno do Recôncavo-TucanoJatobá (Silva, 1988; Camacho, 2017).

Um histórico detalhado dos principais estudos feitos na Bacia do Araripe pode ser encontrado em Ponte \& Ponte Filho (1996).

\section{SENSORIAMENTO REMOTO GEOLÓGICO NA BACIA DO ARARIPE}

\section{Compartimentação tectônica e mapeamento da Bacia do Araripe}

A utilização de sensores remotos na Bacia de Araripe teve início com o surgimento das tecnologias militares de aerofogrametria na década de 1960, dando origem às folhas topográficas lançadas pela SUDENE (1969a, 1969b, 1969c, 1969d, 1969e) e que fundamentaram a intensa produção bibliográfica de mapeamento geológico na bacia a partir da década de 1970, com destaque para os trabalhos de Beurlen (1971) e Mabesoone \& Tinoco (1973) que ao revisar o Grupo Santana (sensu Neumann, 1999) fundamentaram o conhecimento geológico e estratigráfico moderno sobre a bacia.

Os levantamentos aerogeofísicos e geomagnéticos da Bacia do Araripe realizados pela CPRM (Companhia de Pesquisa de Recursos Minerais) a partir da década de 1980 têm sido parte importante no estudo da geologia desta bacia, a princípio do ponto de vista da geologia estrutural, este que elucida os estudos de preenchimento e evolução tectônica da bacia, por tratar-se de uma bacia do tipo Rifte, com destaque absoluto para Ponte \& Ponte Filho (1996), que embasaram o conhecimento sobre a compartimentação estrutural da Bacia do Araripe.

A atual compartimentação estrutural foi proposta por Camacho et. al. (2017) através de dados aeromagnéticos. Nesta publicação foi possível inferir a posição de diversas estruturas ainda não mapeadas, além de fornecer indícios sobre a geometria dos blocos que compõem a bacia e também a processos tectônicos que a originaram, além de tornar mais nítida a segmentação de grábens e horsts da bacia e sua relação com as zonas de cisalhamento presentes no embasamento. O sistema aeromagnético utilizado consiste em um sensor de vapor de césio modelo Geometrics G822-A (tipo stinger) acoplado à cauda da aeronave. As leituras do magnetômetro foram realizadas a cada 0,1 segundo; o que equivale, para a velocidade média de $270 \mathrm{~km} / \mathrm{h}$ da aeronave, a aproximadamente $7,5 \mathrm{~m}$ no terreno investigado (Fig. 2). 

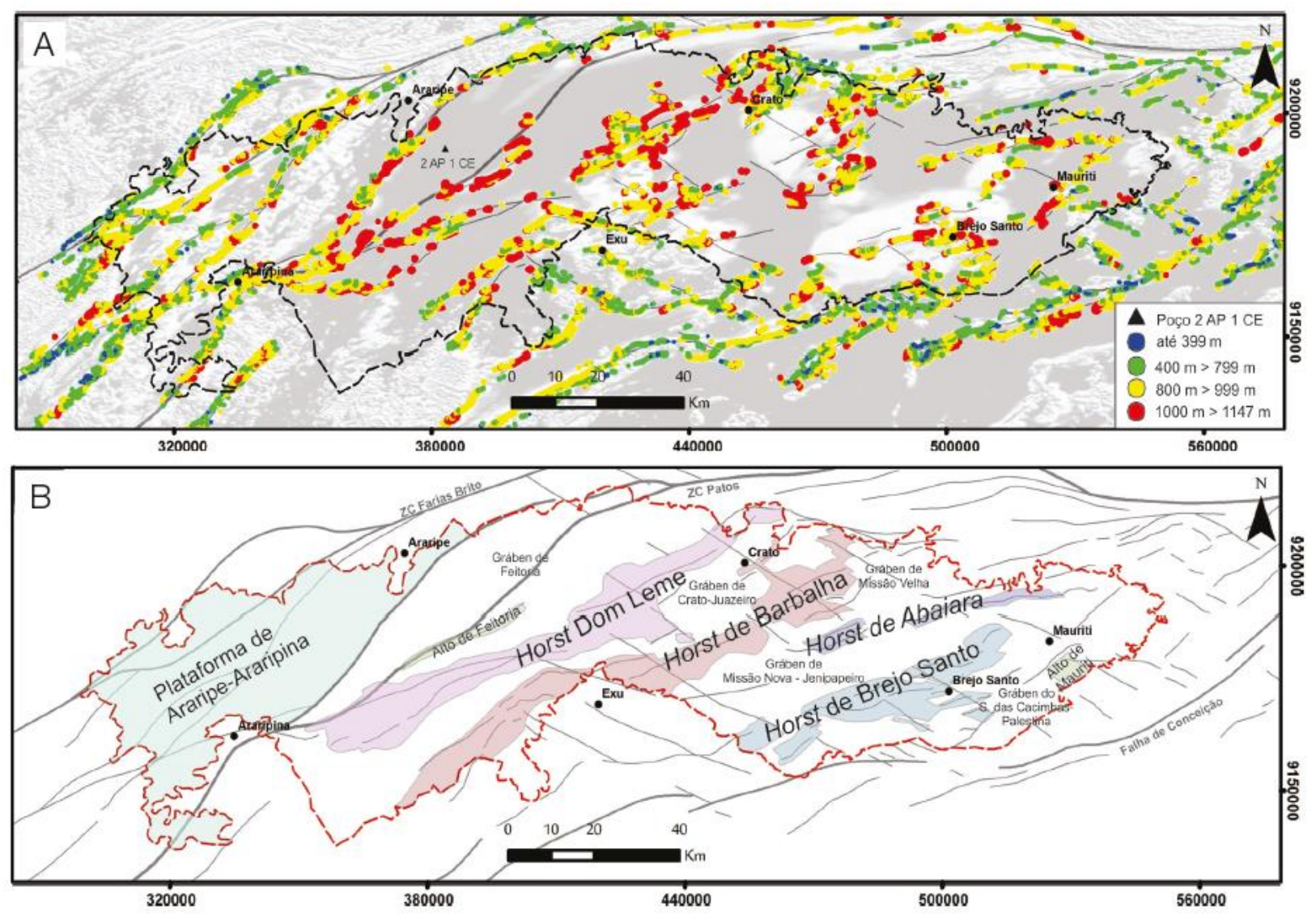

Figura 2 - (A) Mapeamento de estruturas lineares através de anomalias magnéticas levantadas e (B) interpretação do arcabouço estrutural da Bacia Sedimentar do Araripe baseada na identificação dos principais lineamentos magnéticos. (Fonte: Camacho et al., 2017).

No ano de 2017 a CPRM iniciou o mapeamento geológico em escala 1:50.000 da Bacia do Araripe. Os créditos da base cartográfica deste serviço é a Base Planimétrica digital obtida da carta impressa Folha Simões publicada em 1967 pela DSG, ajustada às imagens do Mosaico GeoCover 2.000 de imagens ETM+ do Landsat 7 resultante da fusão das bandas 7, 4, 2 e 8, com resolução espacial de 14,24 metros. Esta base foi editada e atualizada pela Divisão de Cartografia - DICART e pela Superintendência Regional de Recife, através da Gerência de Relações Institucionais e Desenvolvimento GERIDE para atender ao mapeamento temático da CPRM.

Duarte et al. (2017) apresentaram uma metodologia com a utilização de imagens SRTM como ferramenta didática ao Ensino em Geociências Mapeamento de Terrenos Sedimentares, apresentando-as como ferramentas auxiliares na condução e interpretação dos estudos de mapeamento na Bacia do Araripe, considerando-se a interpretação geomorfológica observável neste radar e seu frequente emprego para classificar a superfície terrestre em relação à forma e ao processo que a gerou. Esta utilização de imagens SRTM permite também aplicações ao cálculo de variáveis topográficas, identificação de formas de relevo e estruturas geológicas, potencialmente ocultas em imagens ópticas. 
Modelo tectônico da Bacia do Araripe

Como visto anteriormente $\mathrm{o}$ sensoriamento remoto funciona como uma técnica essencial na caracterização do arcabouço tectônico combinado com dados de campo, estas informações contribuem para o melhor entendimento no processo evolutivo das bacias sedimentares, em especial a Bacia do Araripe.

Marques et al. (2014) compararam dados gravimétricos terrestres e de satélite, além de imagem SRTM e medidas de campo para propor uma compartimentação estrutural com trend diferente daqueles de Ponte \& Ponte Filho (1996) e Assine (2007), além de defender a ocorrência de um processo de inversão durante a evolução tectônica recente da Bacia do Araripe. Marques et. al. (2014) utilizou dados de MDE onde foi observado que topografia principal da Bacia do Araripe é a de um planalto alongado E-W mergulhando suavemente para o oeste. A observação de perfis topográficos mostra uma topografia em degraus aumentando em altitude para o $\mathrm{E}$, onde atinge um máximo de cerca de $1000 \mathrm{~m}$, que resultam do movimento recente ao longo de falhas inversas de direção NW-SE. Estes autores acreditam que a bacia passou por uma fase de inversão tectônica, responsável pela atual topografia da bacia. Isto teria ocorrido após a fase rifte que deu origem a Bacia do Araripe. A interpretação de MDEs foi essencial na identificação das estruturas que os autores classificaram como falhas inversas e que posteriormente foram comparadas aos dados de campo.

Miranda (2015) utilizou imagens de contorno do relevo que foram geradas a partir de dados de altimetria obtidos através de sensor radar, obtidos a partir da missão SRTM. Após a interpolação dos dados foram obtidos mapas topográficos, escala 1:500.000, iluminados artificialmente em quatro direção, com o objetivo de ressaltar as estruturas ortogonais à direção de iluminação escolhida. Miranda (2015) propõe que a bacia passou por dois estágios de evolução tectônica, um do tipo transtensional (caracterizado pela ocorrência de falhas de direção NW-SE) e outro do tipo rifte (caracterizado pela ocorrência de falhas de direção NE-SW).

$\mathrm{O}$ estudo dessas estruturas (lineamentos topográficos) a partir do MDEs mostra que no interior da bacia e no embasamento há dois trends preferenciais de lineamentos, orientados nas direções NW-SE e NE-SW, estes dados são coerentes com o de lineamentos gravimétricos. Os lineamentos NW-SE foram interpretados como falhas normais e/ou falhas transcorrentes, de acordo com o modelo de Riedel, que representam as estruturas nucleadoras do sistema de quebra do embasamento com geometria pull-apart, trama de estruturas mais recente, superimposta às estruturas pretéritas, e que possivelmente originou a formação desta bacia durante o Paleozoico.

Outra ferramenta utilizada na caracterização do arcabouço tectônico é processamento dos dados geofísicos. Carmacho et al. (2017) utilizaram dados aeromagnetométricos para extração de lineamentos magnéticos no interior da Bacia do Araripe, tais lineamentos podem ser associados a estruturas como zonas de cisalhamento, falhas e diques, por exemplo. Paralelamente, por meio do processamento e da interpretação dos dados aerogeofísicos de magnetometria, utilizando filtros de derivadas de primeira ordem nas direções $\mathrm{x}, \mathrm{y}, \mathrm{z}$, possibilitaram a identificação do padrão sigmoidal e as direções de lineamentos magnéticos. A partir da interpretação das derivadas, foi feita a divisão de domínios magnéticos da bacia. Outro método utilizado foi a deconvolução de Euller 3D localizada que proporcionou resultados confiáveis quanto à estimativa de profundidade do topo das principais fontes magnéticas presentes na área de 
estudo, com a vantagem adicional de apresentar uma nuvem de soluções bem menor, em comparação à deconvolução de Euller tradicional (Carmacho et al., 2017). O uso desse dados permitiram inferir a posição de diversas estruturas ainda não mapeadas, além de fornecer indícios sobre a geometria dos blocos que compõem a bacia, os quais estariam fortemente ligados às estruturas presentes no embasamento, como a Zona de Cisalhamento de Patos e a Zona de Cisalhamento Farias Brito, e também a processos tectônicos que deram origem à bacia, segmentando-a gradativamente de oeste para leste, num arranjo complexo de grábens e horsts.

\section{Geomorfologia}

Santos et al., (2009) realizaram um estudo geológico-geomorfológico da sub-bacia leste do Araripe através da aplicação de variáveis morfométricas, altimetria, declividade e formas de terreno em dados SRTM com resolução espacial de $30 \mathrm{~m}$. As representações desses resultados foram geradas no ambiente SIG Idrisi Andes. A importância encontra-se na repercussão que a declividade e formas de terreno têm sobre os processos e dinâmicas geomorfológicos, controlando a morfogênese, pedogênse, corroborando para a definição da morfologia e compartimentação de terreno, logo, fatores que podem ser determinantes para trabalhos geológicos em campo.

Estes autores conseguiram definir as regiões sob influência dos processos de desagregação mecânica, sobretudo desmoronamentos e deslizes por solapamento de base na região estudada, destacando as vertentes limitadas pelo intemperismo na bacia, ou seja, onde taxas potenciais de transporte excedem as taxas de erosão, e a alta produção de detritos nas médias e baixas encostas e incapacidade dos movimentos de massa e fluxos superficiais de remover estes detritos do sistema deposicional. Do ponto de vista da geologia, a maior contribuição dos autores é o reconhecimento morfológico do contato entre a Formação Exu e o Grupo Santana, marcado pela existência de uma escarpa abrupta na primeira, acompanhada de um gradiente suave na jusante dessecados por drenagens e recobertos por sedimentos coluvionares (Santos et al., 2009).

Além da obtenção de informações sobre a geomorfologia e estruturas é possível também obter informações de depósitos minerais a partir da caracterização espectral dos constituintes das rochas, em conjunto com o mapeamento geológico da área de interesse.

$\mathrm{Na}$ região da Bacia do Araripe, Silva et al. (2017) realizaram uma discriminação de gipsita e calcita a partir de imagens do sensor ASTER. A região da Bacia do Araripe apresenta uma intensa produção de gipsita e calcita para a construção civil, devido a isso se faz necessário uma melhor caracterização da área para melhor aproveitamento desses bens. O uso de técnicas ACP (Análise de Componentes Principais), MNF (Minimum Noise Fraction), MTNF (Mixture Tuned Matched Filtering) e SAM (Spectral Angle Mapper) utilizadas no citado estudo foram importantes na separação espectral desses dois minerais, exceto as duas últimas técnicas (Silva et al., 2017).

No sensoriamento remoto, um dos aspectos mais relevantes para estudos de geologia estrutural é a abordagem dentro do âmbito espacial e temporal. Enquanto o primeiro torna possíveis as observações das principais estruturas através da análise dos padrões texturais e formas geomórficas, o segundo permite analisar as variações de suas expressões pelo realçamento sazonal (Liu, 1984). De acordo com o autor, o realce sazonal das feições é o principal motivo do porquê da análise de várias imagens Landsat de diferentes 
épocas fornecer mais informação do que aquela contida numa cena de uma única estação do ano.

\section{Geologia Ambiental}

Accioly et al. (2002) aplicaram transformações espectrais, como os índices de vegetação e a fração de vegetação obtida pelo modelo de análise de misturas em dados do sensor TM/LANDSAT, com o objetivo de se estimar parâmetros estruturais da Floresta Nacional do Araripe, no Ceará. As imagens foram calibradas para radiância, ao nível do solo, e antes do processamento foram removidos seus "outliers". Tanto a fração de vegetação verde quanto os índices de vegetação e as respostas de bandas individuais, apresentaram correlação positiva e significativa com as estimativas da altura média da parcela, da área basal à altura do peito e da área basal na base. Os valores de coeficientes de correlação variaram entre 0,33 e 0,60 . As melhores correlações foram obtidas com o índice de vegetação da razão simples e com o índice estrutural da vegetação para os quais foram estabelecidas as equações de regressão (Accioly et al., 2002).

Sá et al. (2010) analisaram a cobertura vegetal do pólo gesseiro do Araripe (Araripina, Bodocó, Ipubi, Ouricuri e Trindade) com base na classificação não-supervisionada do NDVI e levantamentos de campo. Segundo os autores a análise da cobertura vegetal e uso do solo com NDVI na Região do Araripe Pernambucano foi satisfatória, uma vez que a estatística de Kappa apresentou uma média geral de 0,78 demonstrando uma precisão muito boa para a metodologia aplicada. O mapa de cobertura vegetal e uso da terra que se apresenta neste estudo por ser usado como ferramenta bastante útil para o planejamento do espaço territorial da região da Chapada do Araripe (Sá et al., 2010).

Sá et al. (2011) avaliaram a degradação ambiental no Araripe pernambucano utilizando imagens LANDSAT num intervalo dos anos de 1998 a 2008, que gerou um mapa temático através da classificação das imagens e detecção de mudanças. Segundo os autores, há dificuldade de discriminação da vegetação da caatinga durante o período chuvoso devido à uniformização da cobertura vegetal, concluindo através de sensoriamento remoto multitemporal que a maior causa de degradação severa no Araripe ocorre por conta de atividades agropecuárias.

Silva et al. (2016) apresentaram através da utilização de VANT (Veículo Aéreo Não Tripulado) o cálculo do volume da pilha de rejeito de uma mina de Calcário em Santana do Cariri. Para tanto, realizaram uma modelagem obtida por levantamento GNSS (Global Navigation Satellite System) e LiDAR (Light Detection And Ranging) respectivamente, permitindo a obtenção de informações topográficas tridimensionais da superfície da pilha, inclusive em regiões inacessíveis com precisão, e elevado grau de detalhamento. $\mathrm{O}$ melhor resultado obtido pelo VANT é atribuído principalmente à perspectiva aérea e nadir do levantamento, que permitiu gerar uma nuvem de pontos bem distribuída sobre toda a área de estudo, inclusive entre os pequenos amontoados de material existentes no topo da pilha (Silva et al., (2016). O resultado dos autores não significa que o VANT seja uma ferramenta mais precisa que o GNSS ou LiDAR, mas sim que o VANT foi a melhor solução para o problema proposto, considerando que foram aplicadas metodologias de levantamento de campo que permitissem o melhor resultado no menor espaço de tempo, não sendo possível, por exemplo, realizar um maior número de varreduras com $\mathrm{o}$ 
LiDAR ou um maior caminhamento cinemático com o GNSS, uma vez que a mineração estava em plena atividade.

\section{Hidrogeologia e hidrologia}

Brandão et al. (2007) desenvolveram na porção norte da Bacia do Araripe modelos baseados no alogarítmo CASA, avaliando a evapotranspiração da bacia através da parametrização de fluxos de calor baseada na análise espectral de calor, calculando as reflectâncias de interesse para determinação do NDVI (Normalized Difference Vegetation Index), a fim de estimar o valor da biomassa acumulada na região. Observou-se que para cada tipo de vegetação, ao aumentar o NDVI a biomassa acumulada acima da superfície é incrementada proporcionalmente. Entretanto, os valores médios entre faixas distintas do NDVI são bem diferentes, tendo-se, por exemplo, um aumento de três vezes o valor da biomassa acumulada da vegetação semidensa, com relação à vegetação nativa.

Araújo et al. (2009) utilizaram dados de NDVI do TM Landsat-5 para avaliar impacto ambiental na região da Chapada do Araripe. Os autores determinaram o NDVI e obtiveram os mapas temáticos da sua distribuição espacial. A região foi classificada em área do ponto de vista dos valores dos índices conforme os diferentes tipos de vegetação predominantes na região. Obtiveram três classes: a classe inferior com NDVI < 0,30, a intermediária com $0,30<\mathrm{NDVI}<0,45$ e a superior com NDVI $>0,45$. Numa primeira análise ficou evidenciado que neste período de 21 anos (1987 e 2008) houve um aumento considerável da atividade antrópica nas áreas estudadas (Araújo et al., 2009).

Fernandes et al., (2013) caracterizaram e quantificaram através de dados de imagem Landsat conjugados ao uso de SIG, às mudanças ocorridas na cobertura vegetal e as áreas em risco a desertificação, em parte da Floresta Nacional do Araripe - FLONA, compreendendo os municípios de Barbalha e Crato no Estado do Ceará. Os resultados indicaram que em parte da FLONA entre os anos de 2003 e 2008, ocorreu uma redução da vegetação semidensa a rala, sendo recomposta pela vegetação do tipo semi-densa. Os resultados evidenciam que atenção e vigilância devem ser voltadas para a Floresta Nacional do Araripe, que nesse estudo compreende parte dos municípios de Barbalha e do Crato no Estado do Ceará, em virtude da ocorrência de incêndios, pelos diversos usos agrícolas e pela pressão demográfica em seu entorno, foi observado à ocorrência do nível de degradação grave, mesmo sendo uma área menos expressiva se constitui em uma alerta para os riscos de desertificação (Fernandes et al., 2013).

Silva (2015) estimou a temperatura na microbacia do Rio Granjeiro, na cidade do Crato para o ano de 2011, por meio das imagens do satélite Landsat 5 TM (banda 6), além do mapeamento do uso e ocupação que possam propiciar as diferenças térmicas na microbacia. Para a geração do mapa termal utilizou-se o algoritmo METRIC desenvolvido por Allen et al., (2005). As áreas com temperaturas menos elevadas oscilando entre $21^{\circ}$ e $25^{\circ} \mathrm{C}$, são aquelas que se localizam no topo e encosta da chapada, alto curso do rio, onde apresentam um maior grau de cobertura vegetal e fontes de água. As áreas com maiores temperaturas foram no baixo curso $\left(28^{\circ} \mathrm{C}\right.$ a $34^{\circ} \mathrm{C}$ ), onde a urbanização é mais frequente, e em locais onde o solo encontra-se desnudo (Silva, 2015).

A respeito de trabalhos geofísicos anteriores por eletrorresistividade na região da bacia do Cariri, Marinho et al. (2002), através do estudo de sondagens geoelétricas, caracterizaram os sistemas 
aquíferos da Bacia do Araripe de acordo com suas unidades litoestratigráficas. Este trabalho concluiu que ocorre um aquífero descontínuo raso, com espessura média da ordem de $10 \mathrm{~m}$. Também revelou que a parte inferior da Fm. Exu é constituída predominantemente por sedimentos argilosos, de baixa condutividade hidráulica. Sobreposto ao aquífero, a Formação Exu, em grandes trechos, é formada por arenito duro, por vezes silicificado, que dificulta a construção de poços escavados (cacimbas). A metodologia no método de SEV envolve uma sequência sistemática de coleta de dados em campo e de análise conceitual / computacional desses dados, interpretando-os em forma de estratos geoelétricos horizontais. Os resultados da interpretação de uma SEV são apresentados convencionalmente em forma de gráficos contendo os dados de campo, o modelo com os valores de resistividade impressos e a curva teórica correspondente ao modelo adotado (Marinho et al.,2002).

\section{Estratigrafia}

Para a estratigrafia moderna da Bacia do Araripe podem-se citar trabalhos em que os mapeamentos geológicos foram fundamentados em interpretação geológica de imagens LANDSAT na fase pré-campo. Fambrini et al. (2013) realizaram a análise estratigráfica da Formação Brejo Santo a partir de mapeamento geológico em escala 1:50.000 e dados de afloramentos. Fambrini et al. (2015) propuseram a sucessão de fácies da Formação Barbalha, aliando a descrição dos afloramentos com a interpretação geológica da imagem e de fotografias aéreas da CPRM, e utilizando os mesmos recursos Fambrini et al. (2016) caracterizaram os sistemas deposicional da mesma unidade.

\section{CONSIDERAÇÕES FINAIS}

O emprego de sensores remotos na Bacia do Araripe, utilizam-se do sensor SRTM para estudos geomorfológicos, índices morfomé-tricos, assim como para extração de MDE, bacias hidrográficas e geração de drenagens. As imagens Landsat são utilizadas mais frequentemente para estudos geoambientais e hídricos, destacando-se a utilização das bandas termais para este fim. Observou-se, entretanto, que a integração de imagens de satélite (sistema óptico) e de radar tem sido aplicada na Bacia do Araripe, principalmente, na identificação de estruturas geológicas.

Os métodos não-ópticos geofísicos utilizados na Bacia do Araripe consistem comumente em aerogeofísica de anomalias magnéticas ou gravimétricas, que ressaltam estruturas geológicas do embasamento abaixo de pacotes sedimentares; sondagem elétrica vertical, método que apresenta a estruturação estratigráfica no interior de um pacote sedimentar, apontando anomalias elétricas e consequentes regiões de aquíferos. Alguns destes estudos são complementados com dados de sensoriamento óptico e de radar.

A utilização dos dados do SRTM não deve ser realizada sem uma prédefinição da escala de mapeamento, pois estes dados apresentam imperfeições que não os qualificam para trabalhos que exijam maior rigor na altimetria. Desta forma, os mesmos não permitem que se substitua o uso das curvas de nível presentes nas cartas topográficas. É importante ressaltar que a utilização dos sistemas sensores Landsat e SRTM, pelas suas características de resolução espacial (30m), são adequados para mapeamentos na escala mínima de 1:150.000.

Os conhecimentos apresentados neste trabalho, que envolveram um levantamento de métodos e resultados de publicações científicas, contextualizam técnica e cientificamente o estado da arte 
sensoriamento geológico na Bacia do Araripe.

Espera-se que a abordagem técnico-científica apresentada neste estudo, juntamente com as publicações apresentadas, possam motivar a investigação e a geração de novas pesquisas de sensoriamento remoto geológico na Bacia do Araripe e/ou em outras áreas de estudos geológicos.

\section{REFERÊNCIAS}

Abdullah, A; Akhir, J.M.; Abdullah, I., 2009. A Comparison of Landsat TM and SPOT Data for Lineament Mapping in Hulu Lepar Area, Pahang, Malaysia. European Journal of Scientific Research. Vol.34 No.3, p.406-415.

Accioly, L J. O; Pacheco, A. P., Costa, T. C., Lopes, O. F.; Oliveira, M. A. J., 2002. Relações empíricas entre a estrutura da vegetação e dados do sensor TM/LANDSAT. Revista. Brasileira de Engenharia Agrícola e Ambiental, vol.6 no.3 Campina Grande/PB/Brasil.

Aguiar, R. B., Verissimo, L. S., Colares, J. Q. S., Feitosa, F. A. C., 2006. Evolução do conhecimento geológico e hidrogeológico na Bacia Sedimentar do Araripe. In: XIV Congresso Brasileiro de Águas Subterrâneas, Curitiba, 2006. Anais, 19 p.

Allen, R. G., Allen, M., Morse, A., Trezza, R., 2005. A Landsat-based energy balance and evapotranspiration model in Western US water rigths regulation and planning. Irrigation and Drainage Systmes, v. 19, p. 251-268.

Araujo, A. L., Braga, C. C., Silva, B. B., 2009. "Variação do NDVI na Chapada do Araripe com dados Landsat 5 - TM". In: Simpósio Internacional de Climatologia, Canela - RS, 2009.
Assine, M. L., 1990. Sedimentação e Tectônica da Bacia do Araripe, Nordeste do Brasil. Rio Claro. Dissertação de Mestrado, Instituto de Geociências e Ciências Exatas, Universidade Estadual PaulistaIGCE- UNESP, $124 \mathrm{p}$.

Assine, M. L., 2007. Bacia do Araripe. Boletim de Geociências da Petrobras, Rio de Janeiro. v. 15(2), 371-389.

Beurlen, K., 1971. As Condições Ecológicas e Faciológicas da Formação Santana na Chapada do Araripe (Nordeste do Brasil). Anais da Academia Brasileira de Ciências, v. 43 (Suplemento): 411415.

Brandão, Z. N., Bezerra, M. V. C., Silva, B. B., 2007. Uso do NDVI para determinação da biomassa na chapada do Araripe. In: Anais XIII Simpósio Brasileiro de Sensoriamento Remoto, 2003. 7581.

Camacho, C. R., Oliveira, F. R. F. R., 2017. O arcabouço estrutural da Bacia Sedimentar do Araripe, Província Borborema, baseado em dados aeromagnetométricos. Geologia USP, Série Científica, São Paulo, v. 17, n. 3: 114-161.

Carvalho, T. M., Bayer, M., 2008. Utilização dos produtos da "shuttle radar topography mission" (SRTM) no mapeamento geomorfológico do estado de Goiás. Revista Brasileira de Geomorfologia - Ano 9, no 1.

Carvalho, I .S., Freitas, F.I., Neumann, V., 2012. Chapada do Araripe. In: Hasui Y., Carneiro D. R.C., Almeida F. F. M., Bartorelli A. (org.), Geologia do Brasil. São Paulo: Beca. 2012, v.1, p. 510-513.

Carvalho, T. M.; Latrubesse, E. M., 2004. Aplicação de Modelos Digitais do Terreno (MDT) em Análises Macrogeomorfológicas: 
o Caso da Bacia Hidrográfica do rio Araguaia. Revista Brasileira de Geomorfologia, Uberlândia, v. 4, p. 85-93.

Carvalho, I. S., Melo, J. H. G. 2012. Bacias Interiores do Nordeste. Chapada do Araripe. In: Hasui, I., Carneiro, C.D.R, Almeida, F. F. M., Bartorelli, A. (orgs.) Geologia do Brasil. Editora BECA, São Paulo, p. 502-509.

Carvalho, I. S.; Viana, M. S. S. Lima Filho, M. F., 1993. Os icnofósseis de Vertebrados da Bacia do Araripe (Cretáceo Inferior, Ceará, Brasil). Anais da Academia Brasileira de Ciências, 65(4):459.

Claudino Sales, V., Pelvast, J-P., 2007. Evolução morfoestrutural do relevo da margem continental do estado do Ceará, Nordeste do Brasil. Caminhos de Geografia. v. 7, n. 20 p. 1 - 21, Uberlândia.

Conceição, R. A. C., Silva, A. Q., 2013. Extração automática de lineamentos utilizando imagens SRTM, Landsat ETM+ e ALOS PALSAR na região de Nobres, MT. In: Anais XVI Simpósio Brasileiro de Sensoriamento Remoto - SBSR, Foz do Iguaçu, PR, Brasil, 13 a 18 de abril de 2013, INPE.

Delgado, I. M., Souza, J.D., Silva, L. C., Silveira Filho, N. C., Santos, R. A., Pedreira, A. J., Guimarães, J. T., Angelim, L. A., Vasconcelos, A. M., Gomes, I. P., Lacerda Filho, J. V., Valente, C. R., Perrota, M.M., Heinick, C. A. In: Bizzi, L.A., Schobbenhaus, C., Vidotti, R.M., Gonçalves, J.H. (Eds.), Geologia, Tectônica e Recursos Minerais do Brasil. CPRM, Rio de Janeiro, 2003, 292p.

DNPM, 1996. Projeto avaliação hidrogeológica da Bacia Sedimentar do Araripe. Programa Nacional de Estudos dos Distritos Mineiros. Recife, Departamento
Nacional da Produção Mineral, Distritos Regionais de Pernambuco e Ceará. 101p.

Duarte, C. R., Sabadia, J. A. B., Silva, C. A., 2017. Imagens SRTM: Importante ferramenta didática para o ensino de geociências - o exemplo da Folha Santana do Cariri - Ceará/Pernambuco. Revista de Geologia, Fortaleza, v. 30, n. 1: 7-18.

Fambrini, G. L., Neumann, V. H. M. L., Barros, C. L., Silva, S. M. O. A., Galm, P. C., Menezes Filho, J. A. B. 2013. Análise estratigráfica da Formação Brejo Santo, Bacia do Araripe, Nordeste do Brasil: implicações paleogeográficas. Geologia USP, Série Científica, São Paulo, v. 13, n. 4: 3-28.

Fambrini, G. L., Menezes-Filho, J. A. B., Jesuíno, P. C. L., Araújo, J. T., Durval, L. G. L., Neumann, V. H. M. L., 2015. Sucessão faciológica da Formação Barbalha (Aptiano), Bacia do Araripe, Nordeste do Brasil. Estudos Geológicos UFPE, 25: 139-166.

Fambrini, G. L., Menezes Filho, J. A. B., Neumann, V. H. M. L., Jesuíno, P. C. L., Silvestre, D. C., Lemos, D. R., 2016. Caracterização dos sistemas deposicionais da Formação Barbalha, bacia do Araripe, nordeste do Brasil. Comunicações Geológicas, v. 103, n. 1: 51-65.

Fernandes, F. F., Barbosa. M. P., Moraes Neto. J. M., 2013. Caracterização do Uso das Terras e das Áreas em Riscos de Desertificação em Parte da Floresta Nacional do Araripe (FLONA): Municípios de Barbalha e do Crato, Estado do Ceará. Revista Brasileira de Geografia Física v.06, n.05.14761498.

Grohmann, C. H., Riccomini, C., Steiner, S. S., 2008. Aplicação dos modelos de elevação SRTM em 
geomorfologia. Revista Geografia. Acadêmica, v.2, n.2: p. 73-83.

Hoff, R., Leite, J. C., Zouain, R. N. A., 2010. Aplicação de técnicas de sensoriamento remoto e geoprocessamento como apoio a estudos geológicos e geotécnicos para gestão ambiental do município de três cachoeiras, litoral Norte do RS, Brasil. Embrapa Uva e Vinho-Artigo em periódico indexado (ALICE).

Justo, A.P., 2006. Sistemas orbitais e aéreos aplicados à análise multiescala de lineamentos na borda sudeste da Bacia do Parnaíba. Natal. Dissertação de Mes-trado, Instituto de Geociências, Universidade Federal do Rio Grande do Norte, 65p.

Latrubesse, E. M., Carvalho, T. M., Stevaux, J. C., Moreira, O. L. M., Rodrigues, P. A., 2005. Mapa Geomorfológico de Goiás e Distrito Federal. In: IX Simpósio de Geologia do Centro-Oeste, 2005. Goiânia, página 97.

Liu, C. C., 1984. Análise estrutural de lineamentos em imagens de sensoriamento remoto: aplicação ao Estado do Rio de Janeiro. Tese de doutorado, Pós-graduação em Geociências - USP.157p.

Mabesoone, J. M., Tinoco, I. M., 1973. Paleocology of Aptian Santana Formation (Northeastern Brazil ). Palaeogeography,

Paleoclimatology e Palaeocology, v. 14, n. 2: 87-118.

Madrucci, V., Veneziani, P., Paradella, W. R., 2003. Caracterização geológica e estrutural através da interpretação do produto integrado TM-Landsat 5 e dados aerogamaespectrométricos, região de Alta Floresta - MT, Revista Brasileira de Geofísica, Vol. 21 (3).

Marinho, J. M. L., Cordeiro, W., Vasconcelos, S. M. S., \& Souza,
M. L., 2002. Estudos hidroestratigráficos da Bacia do Araripe, Ceará, utilizando eletrorresistividade. Águas Subterrâneas 1.

Marques, F. O., Nogueira, F. C. C., Bezerra, F. H. R., Castro, D. L., 2014. The Araripe Basin in NE Brazil: an intracontinental graben inverted to a high-standing horst. Tectonophysics, v. 630: 251-264.

Martill, D. M., Wilby, P. R., 1993. Stratigraphy. In: MARTILL, D.M. (ed.) Fossils of the Santana and Crato Formations, Brazil. Palaeontological Association Field Guides to Fossils, 5, p. 20-50.

Matos R. M. D., 1992. The Northest Brasilian rift system. Tectonics, 11(4), 776-791.

Matos R. M. D., 1999. History of the Northeast Brazilian rift system: kinematic implications for the break-up between Brazil and West Africa. In: N.R. Cameron, R.H. Bate, V.S. Clure (eds.). The Oil and Gas Habitats of the South Atlantic. Geol. Soc., London, Spec. Publ., 153, 55-73.

Meneses, P. R., Almeida, T., 2012. Introdução ao Processamento de Imagens de Sensoriamento Remoto. UnB/CNPq, Brasília: 176-179.

Miranda, T. S. D., 2015. Caracterização geológica e geomecânica dos depósitos carbonáticos e evaporíticos da Bacia do Araripe, NE Brasil. Tese de Doutorado, Pós-graduação em GeociênicasUFPE.

Nelson, R. A., 2001. Geologic analysis of naturally fractured reservoirs, 2 nd ed: Boston, Gulf Professional Publishing, 332 p.

Neumann, V. H. M. L., 1999. Estratigrafía, sedimentología, geoquímica y diagénesis de los sistemas lacustres aptiensealbienses de la Cuenca de Araripe 
(Noreste de Brasil). 250 f. Tese

Doutorado em Geologia Universidad de Barcelona, Barcelona, Espanha.

Paradella, W. R.; Bignelli, P. A. ; Venezian, P. ; Pietsch, R.W.; Toutin, T., 1997. Airborne and spaceborne Synthetic Aperture Radar (SAR) integration with Landsat TM and gamma ray spectrometry for geological mapping in a tropical rainforest environment, the Carajas Mineral Province, Brazil. International Journal of Remote Sensing, [S.1.], v. 18, n. 7, p. 483-1501.

Pires, E. F.; Guerra-Sommer, M., 2011. Growth ring analysis of fóssil coniferous woods from early cretaceous of Araripe Basin (Brazil). Academia Brasileira de Ciências, v.83, n.2, p. 409-423.

Ponte, F. C., Ponte Filho, F. C., 1996. Estrutura geológica e evolução tectônica da Bacia do Araripe. Recife: Departamento Nacional da Produção Mineral, $4^{\circ}$ e $10^{\circ}$ Distritos Regionais, Delegacias do Ministério das Minas e Energia em Pernambuco e Ceará.

Restrepo, J. D., López, S. A., 2008. Morphodynamics of the Pacific and Caribbean deltas of Colombia, South America. Journal of South American Earth Sciences, v. 25, n. 1, p. 1-21. doi: 10.1016/j.jsames.2007.09.002.

Sá, I. S.; Galvíncio, J. D., Moura, M. S. B., Sá. I. B., 2010. Cobertura Vegetal e Uso da Terra na Região Araripe Pernambucana. Mercator volume 9, número 19, mai./ago.

Sá, I. I. S., Galvíncio, J. D., Moura, M. S. B., Sá, I. B., 2011. Avaliação da Degradação Ambiental na Região do Araripe Pernambucano Utilizando Técnicas de Sensoriamento Remoto. Revista Brasileira de Geografia Física v. 6, p: 1292-1314.
Santos, C. A., Florenzano, T. G., Nora, E. L. D., 2009. Estudo geológicogeomorfológico da sub-bacia leste do Araripe com aplicação de variáveis morfométricas derivadas a partir de dados SRTM. In: Anais do XIV Simpósio Brasileiro de Sensoriamento Remoto, 2009: 3355-3362.

Silva, J. M. O., 2015. Uso do sensoriamento remoto para a estimativa da temperatura de suerfície na microbacia do Rio Granjeiro/Crato - Ceará. Geosaberes, Fortaleza, v. 6, n. 2: 130-144.

Silva, M. A. M., 1988. Evaporitos do Cretáceo da Bacia do Araripe: ambiente de deposição e história diagenética. Boletim de Geociências da Petrobras, 2(1), 5363.

Silva, M. C., 2017. Caracterização Geológica e Geotécnica dos Debris Flow na Bacia do Córrego Guarda Mão (Itaoca-SP) no Desastre de Janeiro/2014. Dissertação de mestrado, Instituto de geociências da Universidade Estadual de Campinas- Unicamp.

Silva, H. A.; Alencar, M. Lima; Carrino, T. A.; Barbosa. J. A.; Oliveira, P. F. P., 2017. Utilização de imagem multiespectral do sensor ASTER para identificação de calcita e gipsita na Bacia do Araripe - CE. Anais do XVIII Simpósio Brasileiro de Sensoriamento Remoto (SBSR), INPE, Santos SP, Brasil.

Silva, C. A., Duarte, C. R., Souto, M. V. S., Santos, A. L. S., Amaro, V. E., Bicho, C. P., Sabadia, J. A. B., 2016. Avaliação da Acurácia no Cálculo de Volume de Pilhas de Rejeito Utilizando VANT, GNSS e LIDAR. Boletim de Ciências Geodésicas, Curitiba, v. 22, n. 1: 73-94. 
Silva, J. D., Santos, P. R. A. D., 2007. A utilização dos modelos SRTM na interpretação geomorfológica: técnicas e tecnologias aplicadas ao mapeamento geomorfológico do território brasileiro. INPE. In: Simpósio Brasileiro de Sensoriamento Remoto, v. 13, p. 4261-4266.

Souza, M. J. N.; Oliveira, V. P. V., 2006. Os enclaves úmidos e sub-úmidos do semi-árido do Nordeste brasileiro. Mercator, v. 09, p. 85102.

Souza Filho, P. W. M., Paradella W.R. 2002. Recognition of the main geobotanical features along the Bragança mangrove coast (Brazilian Amazon region) from Landsat TM and RADARSAT-1 data. Wetlands Ecology and Management, 10: 123-132.

Souza Filho, P. W. M.; Paradella, W. R., 2003. Use of synthetic aperture radar for recognition of Coastal Geomorphological Features, landuse assessment and shoreline changes in Bragança coast, Pará, Northern Brazil. Anais da Academia Brasileira de Ciências, v. 75 , n. 3, p. 341-356.

SUDENE, BRASIL. Ministério do Exército. DSG. Fronteiras. Rio de Janeiro, 1969a. 62 × $74 \mathrm{~cm}$. Folha SB.24-Y-C-III. Escala 1:100.000. $7^{\circ} 00^{\prime}-7^{\circ} 30^{\prime} \mathrm{S}, 40^{\circ} 30^{\prime}-4100^{\prime} \mathrm{W}$.

SUDENE, BRASIL. Ministério do Exército. DSG. Campos Sales. Rio de Janeiro, 1969b. 62 × $74 \mathrm{~cm}$. Folha SB.24-Y-D-I. Escala $1: 100.000 .7^{\circ} 00-7^{\circ} 30^{\prime} \mathrm{S}, 40^{\circ} 00-$ $40^{\circ} 30^{\prime} \mathrm{W}$.
SUDENE, BRASIL. Ministério do Exército. DSG. Santana do Cariri. Rio de Janeiro, 1969c. 62 x 74 cm. Folha SB.24-Y-D-1l. Escala 1:100.000. $7^{\circ} 00^{\prime}-7^{\circ} 30^{\prime} \mathrm{S}, 39^{\circ} 30^{\prime}$ $40^{\circ} 00^{\prime} \mathrm{W}$.

SUDENE, BRASIL. Ministério do Exército. DSG. Crato. Rio de Janeiro. 1969d. 62 x $74 \mathrm{~cm}$. Folha SB.24-U-II. Escala 1:100.000. $7^{\circ} 00^{\prime}-7^{\circ} 30^{\prime} \mathrm{S}, 39^{\circ} 00^{\prime}-39^{\circ} 30^{\prime} \mathrm{W}$.

SUDENE, BRASIL. Ministério do Exército. SGE. Simões. Rio de Janeiro, 1969e. 62 x $74 \mathrm{~cm}$. Folha SB.24-Y-C-VI. Escala 1:100.000. $7^{\circ} 30^{\prime}-8^{\circ} 00^{\prime} \mathrm{S}, 40^{\circ} 30^{\prime}-41^{\circ} 00^{\prime} \mathrm{W}$.

Strugale, M., Rostirolla, S. P., Mancini, F., Portela Filho, C.V., Ferreira, F. J. F., Freitas, R. C., 2007. Structural framework and Mesozoic-Cenozoic evolution of Ponta Grossa Arch, Paraná Basin, southern Brazil. Journal of South American Earth Sciences, 24: 203227.

Teixeira, S. G.; Souza Filho, P. W. M., 2009. Mapeamento de ambientes costeiros tropicais (Golfão maranhense, Brasil) utilizando imagens de sensores remotos orbitais. Revista Brasileira de Geofísica, v. 27, p. 69-82.

Veríssimo, L. S., Aguiar, R. B., 2005. Hidrogeologia da porção oriental da bacia sedimentar do Araripe. Meta A. Diagnóstico do estado da arte. Brasil: CPRM. Disponível em:

$<$ http://www.cprm.gov.br/publiqu e/media/araripe_meta_A.PDF>. Acesso em: 15 abr. 2015. 